\title{
SYSTEMATIC ERRORS IN THE PHOTOGRAPHIC POSITIONS \\ OF DOUBLE STARS AT THE SPROUL OBSERVATORY
}

\author{
SARAH LEE LIPPINCOTT \\ Sproul Observatory, Swarthmore College, Swarthmore, Pa., U.S.A.
}

The long range program of the Sproul Observatory initiated in 1937 by Peter van de Kamp has been primarily devoted to a detailed astrometric study of the positions of nearby stars from photographs taken with the 24-in. refractor. The accumulation of plates over a long time interval provides the opportunity for finding perturbations of small amplitude leading to the discovery of unseen companions. By now there are $30 \mathrm{yr}$ of observation on most of the stars with parallaxes greater than +0.10 and brighter than the 12 th magnitude, which are observable at latitude $+40^{\circ}$.

The stars studied include both classical doubles and many designated single. Some of these show variable motion which can be interpreted as Kepler motion. For binary components well within $2^{\prime \prime}$ of each other their blended photographic image is the photocenter which revolves about their center of mass. If the difference in magnitude is greater than 3, the photocenter is essentially the position of the brighter star, and one observes the brighter star's orbit about the center of mass of the system; the amplitude of this orbit is only a small fraction of the amplitude of the relative orbit.

In order that stars be not known previously as doubles, their companions must be fainter by several magnitudes and their separations generally less than a second of arc. (Those having far less separation would have very short periods and would be within the realm of spectroscopic binary discovery). The limits given above depend on the facility of visual detection. Programs for visual discovery of close binaries among

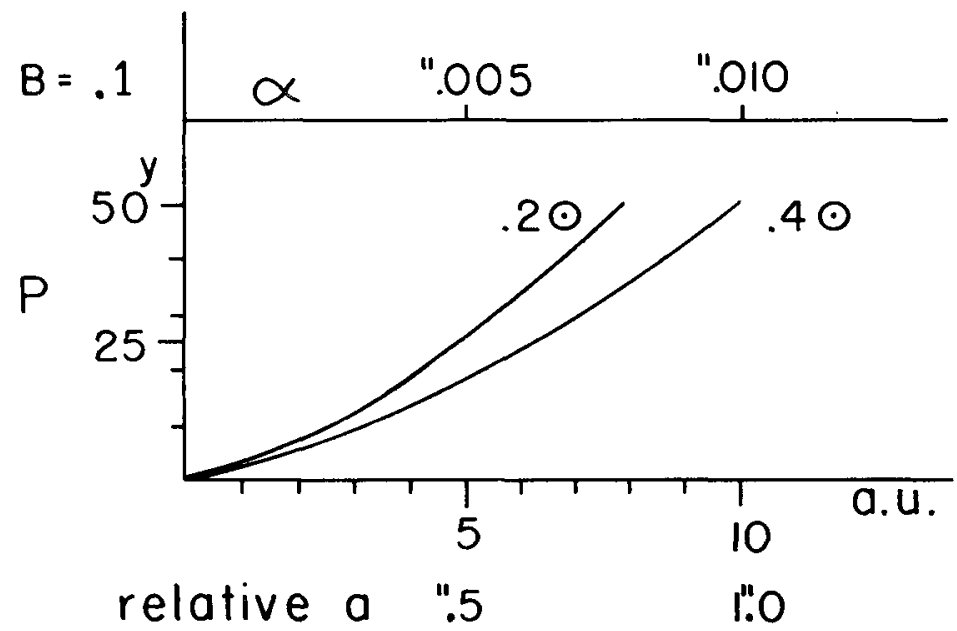

Fig. 1. Scales of orbits for systems of various periods with total masses of $0.2_{\odot}$ and $0.4_{\odot}$. The relative $a$ and $\alpha$ hold for $\pi=+0^{\prime \prime} .1$. 
nearby stars have been undertaken, for example by Kuiper (1935); however, they must be extended over long time intervals so that the systems may be observed at times of greatest separation.

Most of the stars on the Sproul program are red dwarfs possessing masses around $0.3_{\odot}$ or smaller. Figure 1 gives some indication of the expected amplitudes with respect to values for the period; the values in seconds of arc are for $\pi=+0$." 1 . Red dwarfs are considered with the sum of masses being $0.2_{\odot}$ and $0.4_{\odot}$. If $\Delta m$ is large and the mass of the faint component is 0.1 of the total mass of the system, $\alpha$ represents semi-axis major of the visible component around the center of mass. One sees that the amplitude may be very small and consequently one must pay strict attention to systematic errors in the recorded positions, especially to those which are of a seasonal nature or persist over several years.

In order to achieve the desired precision the mean of many observations must be taken during one or more seasons. For example, Peter van de Kamp has obtained about 100 plates ( 4 per night) per year on Barnard's star; for less privileged stars there may be only 20 plates per year on the average. With good images one should expect a precision of about $\pm 0^{m m} .0005$ p.e. for a yearly mean position. (The p.e. of unit weight is about $\pm 0^{m m} .0020 ; 1 \mathrm{~mm}=18$."87.) Because of systematic seasonal errors, we cannot realize the theoretical accuracy given by the sum of the weights.

Recently we have studied the systematic errors by taking the residuals from least squares solutions of individual stars, compiling them according to epoch of observation and then grouping them with respect to position in the sky. The 'composite star' mean residuals in $X$ and in $Y$ can show only systematic errors and nothing of cosmical interest. The stars which appear to be of interest from the distribution of the residuals are compared with the 'composite star' residuals in the same part of the sky. A comparison may give some confidence in the reality of small amplitude perturbations. The mean residuals of stars in the region of the sky of Barnard's star show no systematic errors with respect to time of observation (Figure 2). On the other hand the region of $\mathrm{BD}+20^{\circ} 2465$ is suspect. $\mathrm{BD}+20^{\circ} 2465$ is a red dwarf which gave indication of a perturbation in 1936 from observations made at the McCormick Observatory (Reuyl, 1943). After $30 \mathrm{yr}$ observations made at Sproul, which show non-random yearly residuals, there are difficulties in explaining the deviations from linear proper motion in terms of Kepler motion. In view of the systematic errors in that part of the sky one must be very careful in interpreting the positions of $\mathrm{BD}+20^{\circ} 2465$.

The amplitudes of the perturbations already published at the Sproul Observatory range in size from $0^{m m} .0011$ to $0^{m m} .0230$ in $X$ and $0^{m m} .0006$ to $0^{m m} .0385$ in $Y$. There are other suspected perturbations which require further observations to confirm their interpretation in term of Kepler motion. Their amplitudes range from $0^{m m} .0015$ to $0^{m m} .0090$ in $X$, and $0^{m m} .0015$ to $0^{m m} .0100$ in $Y$. Figure 3 shows the distribution of the amplitudes in $X$ and in $Y$.

The likely masses for the non-visible companions range from $0.4_{\odot}$ down to $0.0012_{\odot}$ for the mass of Barnard's star B2. The masses of the visible stars whose companions have been revealed by the above method are between $3_{\odot}$ and $0.15_{\odot}$. 


$$
V_{x}
$$

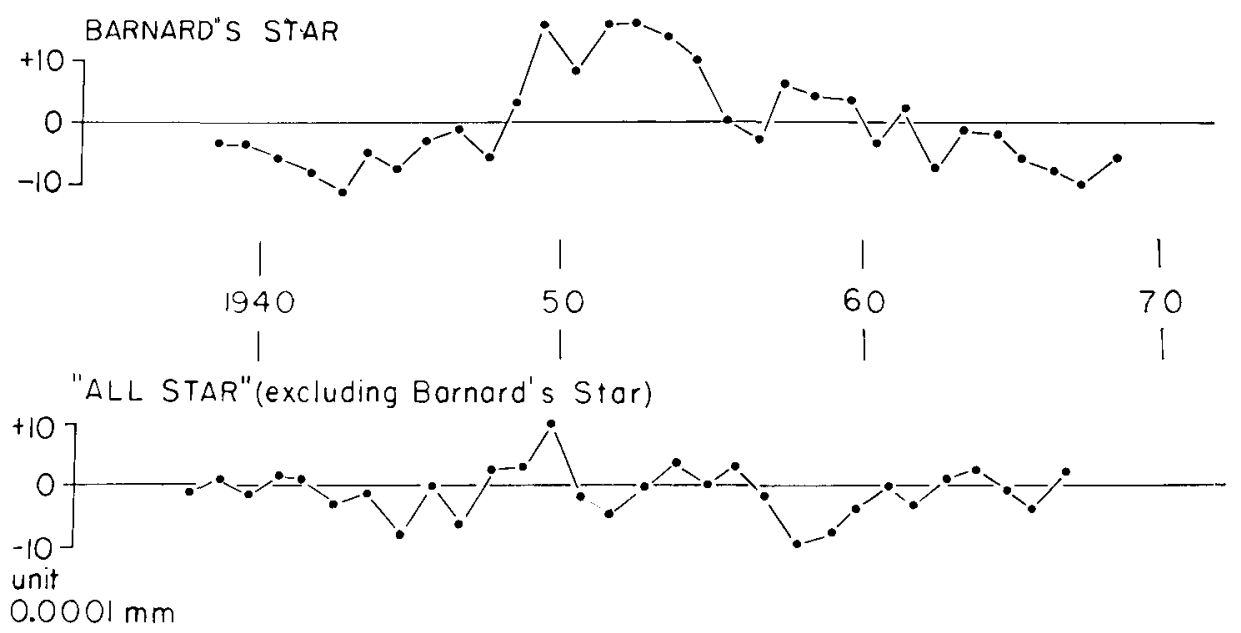

Fig. 2. Above: Barnard's star yearly mean residuals in $x$ from general least squares solution. Below: 'All Star' residuals in $x$ from low declination group excluding Barnard's star.

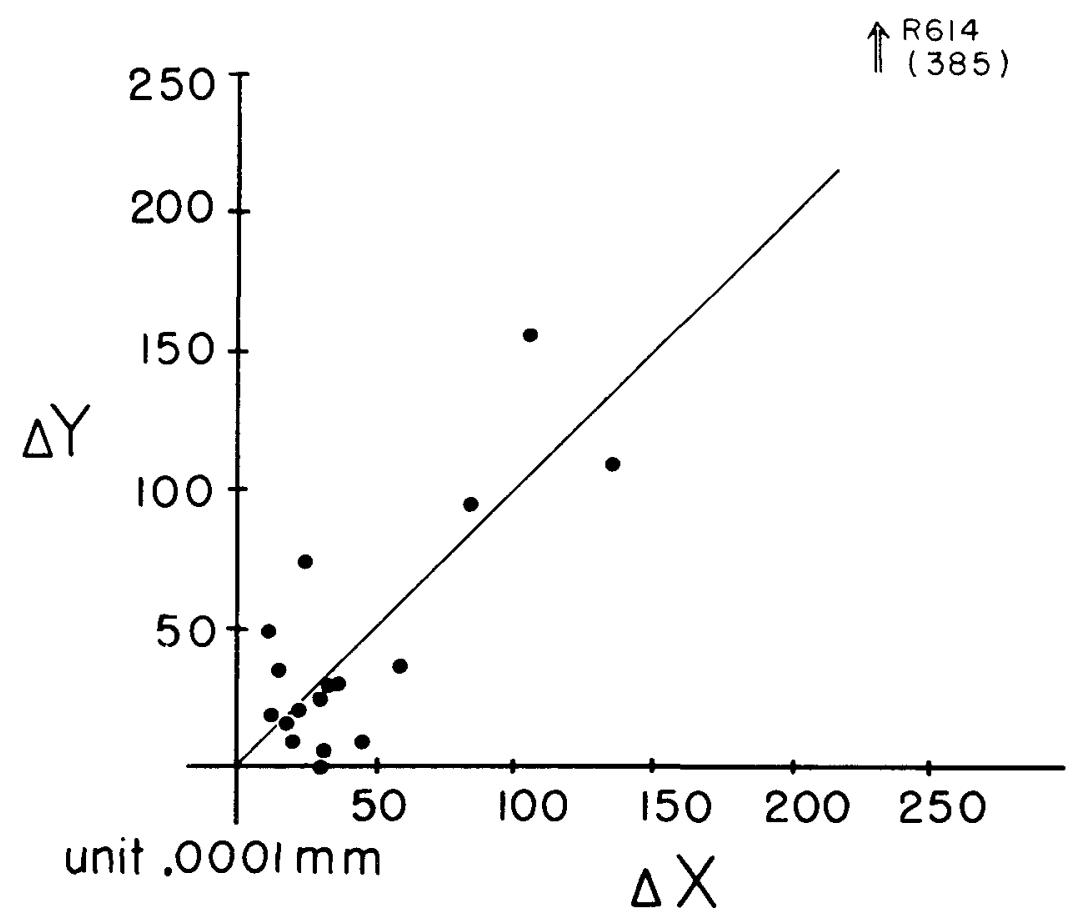

Fig. 3. Sproul observations of maximum $\Delta X$ and $\Delta Y$ indicating unseen companions. Ross 614 , whose companion was later seen, is also shown. 
Another problem which concerns the photographic positions of double stars is the position of the blended photographic image for a known double. Pairs which are separated by less than 2 sec of arc generally appear blended on the Sproul photographs. The question has long been asked - what is the position we observe, where is it with respect to the positions of the two components? The problem has been studied by Hall $(1949,1951)$ and by Duke $(1954,1955)$ with experiments on the blending of artificial images. Taking the theoretical point of view, Pierre Morel, while at the Sproul Observatory and more recently at the Observatoire de Nice, has evaluated the position

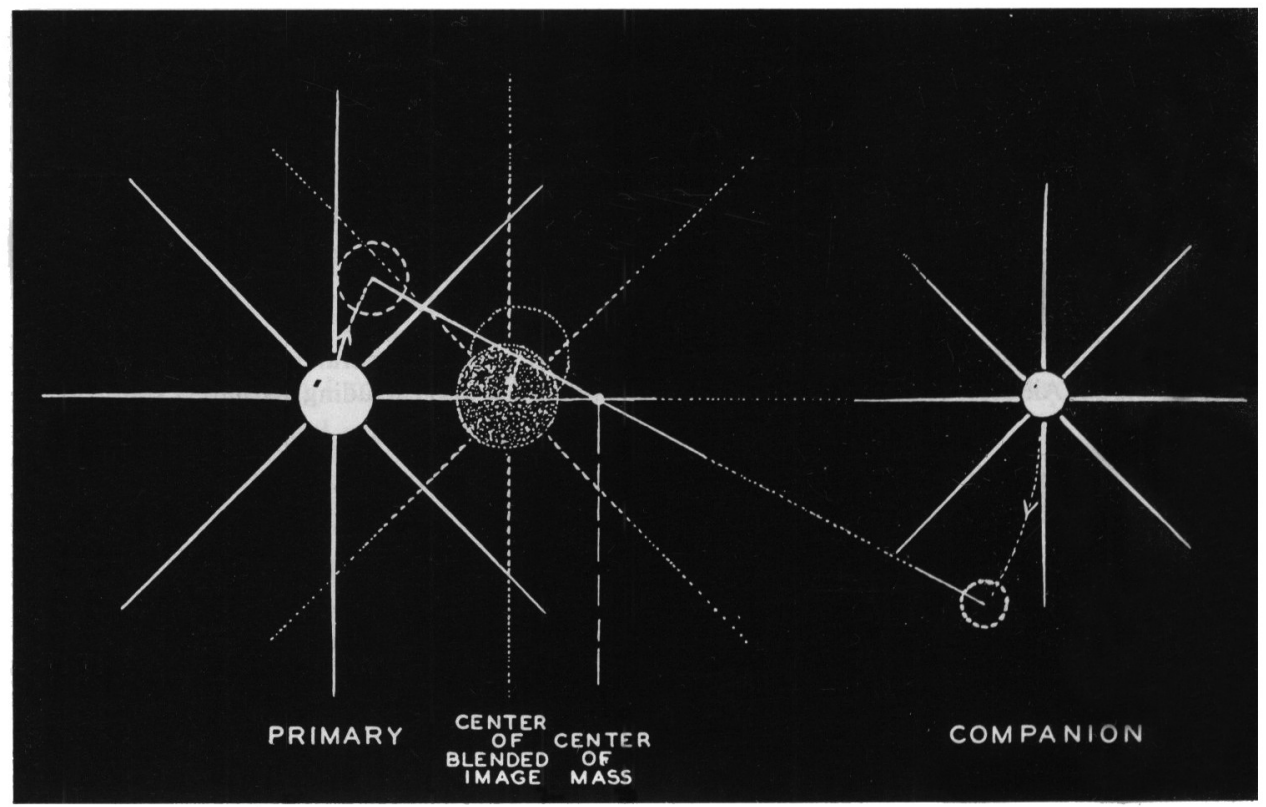

Fig. 4. Relative positions of photocenter and barycenter with respect to primary and companion.

of blended images. During the past year Feierman (1971) has used a new observational approach at the Sproul Observatory.

The position of blended images has been defined as the center of luminosity, and is related to the positions $A$ and $B$ as shown in Figure 4 by

$$
\beta=\frac{l_{B}}{l_{A}+l_{B}},
$$

where $l_{A}$ and $l_{B}$ are the luminosities of $A$ and $B$ taken at their effective wavelengths. Feierman has devised an interesting method for evaluating the blending effect on position. A coarse grating in front of the 24-in. Sproul objective produces diffraction pattern images on either side of the central image. By orienting the grating at the proper angle and photographing properly chosen wide visual double stars blended images are formed whose individual image positions and $\Delta m$ are obtained from de-orienting the 

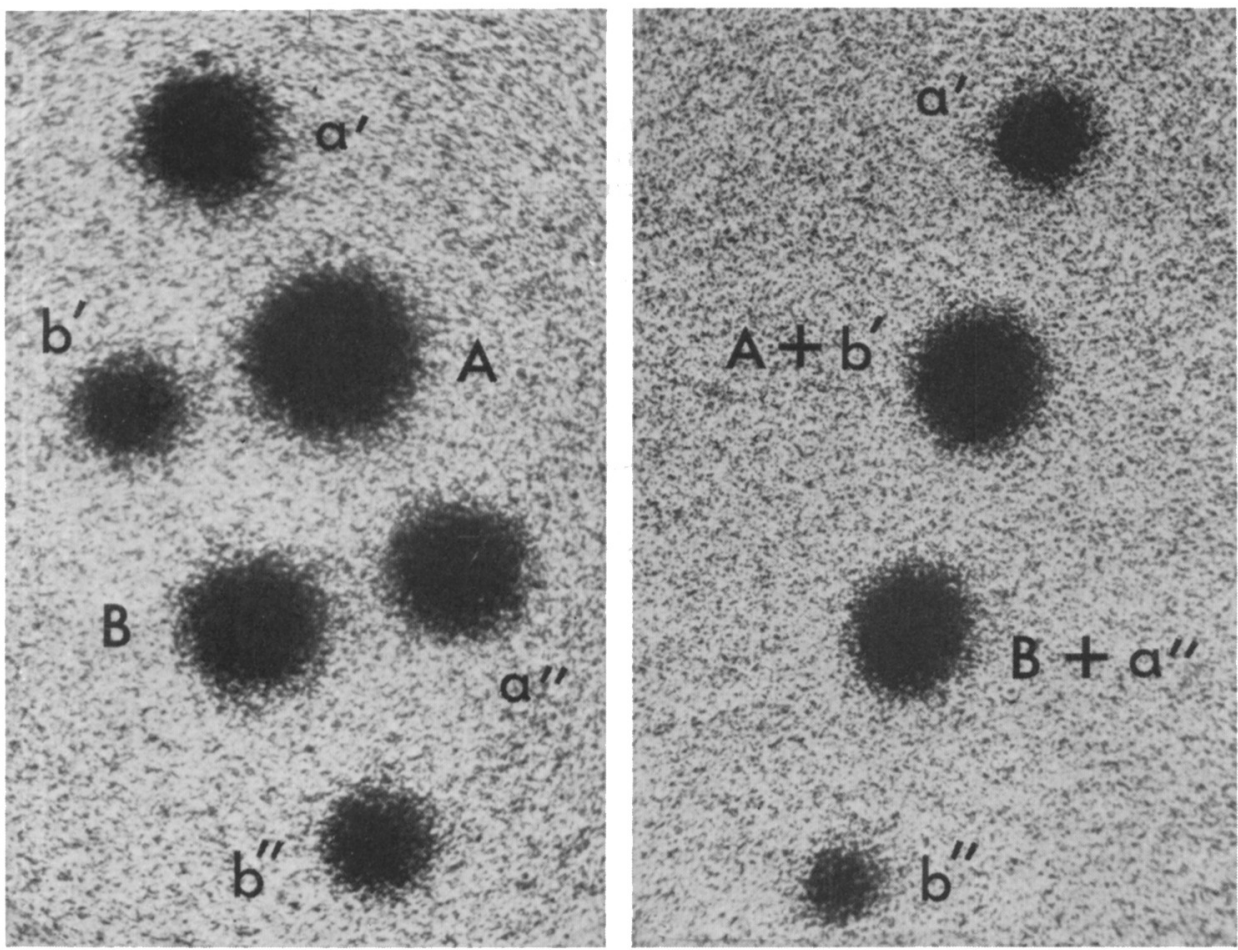

Fig. 5. Two photographs of the double star $\Sigma 2452$ taken with the Sproul 24-in. refractor and a coarse objective grating. Left: $A$ and $B$ companions flanked by 1 st order images 1 magnitude fainter than central images. Right: Grating oriented so that images overlap to simulate blending of close double star images. (Photographs by Barry Feierman.)

grating and again measuring the spacing of the component images (Figure 5). Feierman concludes from his study that the weighted center of light given by the above formula holds for $\Delta m<1^{m} .5$ while for greater values of $\Delta m$ the value for $\beta$ falls off more rapidly confirming the results of Hall, Duke, and Morel. For separations greater than $0.05 \mathrm{~mm}$ he concludes that the $\beta$ is dependent on exposure time and the diameter of the composite image.

\section{References}

Duke, D.: 1954, Astron. J. 59, 184.

Duke D.: 1955, Astron. J. 60, 42.

Feierman, B.: 1971, Astron. J. 76, 73.

Hall, R. G.: 1949, Astron. J. 54, 106.

Hall, R. G.: 1951, Astron. J. 55, 212.

Kuiper, G. P.: 1935, Publ. Astron. Soc. Pacfic 47, 230.

Reuyl, D.: 1943, Astrophys. J. 97, 186.

\section{Discussion}

Strand: What is the rms for the 'composite star' average in the region near Barnard's star? If the variation is assumed to be systematic errors, and is applied to the Barnard star perturbation, what is the effect upon it? 
Lippincott: As you can see from the slide the yearly mean residuals from the 'composite star' are generally under $\frac{1}{2} \mu$. The Barnard's star residuals from the orbital solution are slightly improved if adjusted by the 'composite star' residuals.

Van de Kamp: Applying the correction as given by the 'composite star' for low latitudes, the perturbation remains, and is not changed materially. I have not yet published a 'corrected' result since it has been my policy not to apply systematic corrections unless they are very accurate. Otherwise they may do more harm than good. 